\title{
Precision Medicine and Non-Colorectal Cancer Liver Metastases: Fiction or Reality?
}

\author{
Effie Liakopoulou ${ }^{a}$ Alexander Knuth ${ }^{b}$ \\ a Division of Hematology, University of Washington, Seattle, WA, USA, \\ ${ }^{b}$ National Center for Cancer Care \& Research, Cancer Services, Hamad Medical Corporation, Doha, Qatar
}

\section{Keywords}

Liver metastases · Non-colorectal cancer .

Precision medicine $\cdot$ Immune oncology $\cdot$ Clinical trials

\section{Summary}

Background: Non-colorectal liver metastases (nCRLM) constitute a variety of heterogeneous diseases and a considerable therapeutic challenge. Management is based on the primary tumor and the clinical course. In the era of precision medicine (PM) we know that cancer is heterogeneous within the tumor and across different sites. Methods: We give an overview of the path to PM through 'omics' beyond genomics. We refer to the experience gained to date from models such as colorectal cancer and we discuss the opportunity offered by PM for the management of nCRLM. Results: In order to best characterize and track tumor biological behaviors as well as to understand mechanisms of response to therapy and survival we suggest the application of novel clinical trial designs, a dynamic approach with serial monitoring involving evaluation of primary and metastatic sites. Quality and standardization of tissue acquisition and biobanking is a precondition for the reliability of this approach. Conclusion: The application of PM is increasingly becoming a reality. Elucidating the mysteries of tumors in complex settings can only be achieved with the approach PM offers. nCRLM may serve as a model for the application of PM principles and techniques in understanding individual diseases and also cancer as an entity and therapeutic challenge.

(C) 2015 S. Karger GmbH, Freiburg

\section{Introduction}

Since Hippocrates (460-370 BC), medicine has retained its aspiration of being personalized $[1,2]$. In the past 500 years, advances towards personalized medicine have been made in an ever shorter time span, starting with Vesalius's contribution to anatomy [3], to Virchow's introduction of modern pathology [4], and up to 'omics' technologies of the last decades [5]. Following the genetics revolution coinciding with a paradigm shift in information technology, our ability to gain insight and understanding of the biology of human diseases has greatly accelerated - and in turn, our ability to further advance our approach towards personalized medicine.

These more recent technological developments have provided us with a new set of tools in precision medicine (PM)/oncology. This has triggered significant advances in disease assessment, management, and prevention. Additionally, biobanking and the standardized assertion of biological material are now a key precondition for generating reproducible results to enable precision assessment of the individual patient's disease. Developments in methods of PM now challenge traditional classification and evidence-based management of disease and of cancer in particular. However, PM has still to overcome significant hurdles in operational, regulatory, and logistical aspects of patient treatment. An imminent replacement of traditional approaches is therefore unlikely. Thus, the current situation is best described as a transition period from traditional evidence-based methods to one of a personalized, participatory, and predictive approach based on precision.

Models and experience gained from the initial application of personalized medicine in e.g. colorectal cancer (CRC), breast cancer, and melanoma suggest the feasibility of personalized management in even more complex modalities including metastatic liver diseases.

\section{KARGER \\ Fax +497614520714

\section{() 2015 S. Karger GmbH, Freiburg}

$1662-6664 / 15 / 0316-0434 \$ 39.50 / 0$
Prof. Dr. med. Alexander Knuth

Cancer Services, Hamad Medical Corporation

P.O. Box 3050, Doha, Qatar

KKnuth@ hamad.qa 


\section{Precision Medicine and Personalized Approach to Illness}

In his 2015 State of the Union Address President Obama called on the USA and the world to adopt a new era of medicine that '... brings us closer to curing diseases like cancer and diabetes, and to give all of us access to the personalized information we need to keep ourselves and our families healthier ...' [6]. His call coincides with a number of other national efforts to consciously embrace this approach to medicine. These developments herald the era of PM [7].

The US National Cancer Institute defines personalized medicine as '.. a form of medicine that uses information about a person's genes, proteins, and environment to prevent, diagnose, and treat disease ...' In cancer, personalized medicine uses specific information about a person's tumor to help the diagnosis, plan treatment, assess the effectiveness of treatment, or define the prognosis [8], hence the term PM. However, PM and precision oncology in particular are not restricted to the recent genome-related technologies only. Notably, with the discovery of blood group antigens PM started to mark its defined place in modern management of health and disease more than a century ago.

$\mathrm{PM}$ with the potential for more detailed and precise diagnosis and identification of disease biology, treatment options tailored to the specific patient resulting in fewer adverse side effects, promises a significant impact on medical practice. The participatory and preventive approach of personalized medicine offers patients an incentive to consciously adapt their lifestyle, e.g. to manage identified genetic susceptibilities that may lead to disease.

In this new era the approach to cancer as an entity and the research involved demands a reassessment and change of culture in medicine [9].

With the proper regulatory and legal framework in place we will be able to engage our patients in the diagnostic and therapeutic decision making process and to counsel families and individuals on genetic risks and prevention of disease. What is still research today may rapidly become routine clinical management tomorrow. Already the considerable investment into the future of PM is starting to pay off for itself in many ways with some dramatic changes in therapeutic approaches and outcomes [10-12]. The regulatory approval process for new treatments resulting from developments in PM research should be revisited to contain costs and time loss as a major hindrance to medical progress [13].

\section{From the Glycome, Proteome, and Immunome to the Genome and Microbiome: Paving the Road of Medicine to Precision with omics}

The completion of the Human Genome Project is among the significant advances in medicine over recent decades. This, combined with the technological evolution in analysis, processing, and mapping of genetic information and advances in the processing power of information technology and bioinformatics, has driven medical care into the era of personalized medicine. As an example, the construction of genomic haplotype maps enables the visualization of DNA in a much more precise picture than ever $[14,15]$.

Spectacular recent advances cannot overshadow, however, that PM came to bear with the discovery of blood group antigens by Landsteiner [16] in 1900. It is with this discovery and the evolution around molecular medicine that diagnostic or therapeutic targets were identified, including glycosylation variants of some secreted blood group antigens as tumor markers like Sialyl-Lewis ${ }^{\mathrm{A}}$ or carbohydrate antigen (CA) 19-9 [17].

The search for human cancer antigens by glycome and proteome research has uncovered diagnostic and therapeutic markers like carcinoembryonic antigen (CEA), growth factor receptors such as the epidermal growth factor receptor (EGFR) family, and others which are now essential components for patient assessment, treatment, and prognostic evaluation.

With the dissection of the human cancer immunome, e.g. by typing tumors with autologous sera and in particular by the SEREX (serological expression cloning) technology, as pioneered by Old et al. $[18,19]$, multiple families of human cancer antigens were uncovered as targets for immunotherapy and immune intervention. With the recent discoveries around the clinical potency of immune checkpoint blockade of co-modulatory molecules like CTLA-4 or PD-1, the immunome may evolve as a significant biomarker of response in combination with checkpoint blockade. The pre-existing immunity to cancer antigens may predispose to a better response to checkpoint blockade than without any detectable cancer immunity [20].

Multiple molecular targets for therapeutic intervention (KRAS, ALK, BRAF etc.) have been identified over the past two decades, and this has ushered in an era of molecular medicine with unprecedented magnitude and impact. More recently, the exploitation of the mutanome of a given tumor or patient has been addressed in clinical research to develop therapeutic strategies to target individual mutations. Pre-existing immunity to mutanome targets appears to constitute a predisposing and predictable setting for a response to immune checkpoint intervention [21].

On the one hand, the influence of the microbiome in the gastrointestinal tract is recognized to play a major role for the response of a patient's immune system to cancer antigens and to immune function. On the other hand, conventional treatment modalities like cytotoxic chemotherapy or antimicrobial therapy may influence the microbiome itself and alter its systemic impact, guiding our therapeutic strategies in the future [22,23].

Finally, omics have and still are leading us to a better understanding of therapeutic modalities like ionizing radiation and chemotherapy, in that they may not only directly control tumor growth but also indirectly induce immunostimulatory effects like complement activation and upregulation of HLA molecules and cancer antigens facilitating immune control of cancer [24-27].

Dissecting the genome at different levels is still a matter of intensive research and discovery for disease assessment and management in the routine setting and for basic research. However, where in the routine setting biopsies of whatever kind may become available, proper biobanking facilities and standards must be in place to 
be able to pursue open or future research questions under proper conditions.

PM shaped the 20th century and will continue to shape medical research and treatment in the 21 st century. The assessment of noncolorectal liver metastasis (nCRLM) should be seen in the context of a bigger picture. The principles of PM can be nicely displayed in this setting, applying 'omics' technologies for established, experimental, and still to be discovered targets in the future.

\section{Personalized Approach to Metastatic Liver Disease: The Evolving Role of Precision Medicine}

The liver is the most common site of metastases which are thought to hematogenously spread from a variety of tumors. Often, isolated hepatic metastases are associated with CRC, and in these cases the extent of liver involvement is related to survival. Surgical resection of the metastatic site has traditionally offered the only potential of cure for CRC metastatic disease. Less frequently, hepatic metastases are associated with other cancers including breast, melanoma, gastrointestinal, neuroendocrine, and other tumors [22, 23].

Metastases from carcinoid and islet cell tumors usually show a slow rate of growth, often resulting in prolonged survival even in the case of bilobar or multicentric liver disease [28-33]. Only few cases with liver metastases due to non-colorectal tumors present with resectable disease and are limited to the liver. Therefore, in these cases systemic therapy is the primary treatment option often with less favorable outcome.

For the management of hepatic metastases from non-CRC primary tumors, individualized treatment approaches are recommended, based on the origin and nature of the primary tumor, the patient's clinical course including symptoms, and the extent of the disease [34]. Advances in the understanding of liver diseases and liver cancers in the last decades include the application of new technologies to identify molecular abnormalities which may provide an even more accurate classification of disease and stratification of treatment options for patients diagnosed with liver cancer. This includes e.g. the discovery of the TP53 hotspot mutation [35, 36] assisting the classification and treatment decision by using conventional clinical criteria in combination with molecular information. In parallel, the advancement of imaging methods has contributed to earlier diagnosis, accurate staging, and treatment advances using structural and molecular imaging. In addition, radiogenomics relates imaging of biomarkers to these genetic and molecular features [37]. The result is that several treatment options are now available with proven survival benefit dependent on proper selection and application [38].

Personalized medicine is often discussed in the context of targeted therapies including biological, hormonal, and immune therapies. However, chemotherapy has also the potential to be tailored to individual patients. While biomarker research has mainly focused on targeted therapies, there have been continuous efforts to identify predictive markers of response or resistance to chemotherapy.
In recent years, diagnostics and predictive biomarkers have been developed for CRC and other tumors prone to metastasis to the liver. Individual disease profiling by molecular and imaging technologies may offer strategies to improve outcomes in combination with resection, adjuvant, regional, or systemic chemotherapy, and targeted treatments.

At this time, morphologic classification of disease still is a diagnostic fundament. Modern pathology includes immunopathology, cytogenetics, and molecular pathology for individual disease assessment and identification of new treatment options $[39,40]$. Next generation sequencing (NGS) has provided insights into cancer biology. It is now accepted that each cancer is unique and arises from a distinct combination of genetic alterations [41]. Cancers, even within individual patients, present remarkable heterogeneity and dynamic response to selective treatment pressure with resistant subclones expanding [42-45].

- The example of metastatic colorectal cancer ( $m C R C)$ : The recent developments in CRC treatment constitute one of the best examples of how increased understanding of molecular biology combined with novel diagnostic techniques and therapeutic advances can guide tailored treatment regimens and optimize outcomes [46]. In addition, CRC is an example for the personalization of treatment outside of the context of targeted therapies as well as within. Irinotecan, traditionally used for the treatment of CRC, was one of the first chemotherapy agents to be tailored to the recipient's needs according to the recipient's pharmacogenomics profile $[47,48]$. In addition, and despite unavailability of a standard test for its assessment, the expression of excision repair cross-complementing $\mathrm{C} 1$ (ERCC1) is now the most promising predictive marker for resistance to oxaliplatin [49]. It has also been suggested that oxaliplatin-related neurotoxicity may be a result of genetic differences identified in the recipients' metabolic pathways [50]. Furthermore, highly accurate prediction of response to FOLFOX (folinic acid, 5-fluorouracil, and oxaliplatin) based on gene expression profiles is warranting its implementation in selecting patients for treatment [51]. To develop targeted therapies, understanding of the molecular mechanisms of CRC led to the development of monoclonal antibodies (mAbs) against factors in key carcinogenic pathways. These involve widely used agents targeting angiogenesis and EGFR signaling. With the development and availability of a variety of effective therapies the need for predictive biomarkers increased. This enables optimum treatment selection for each patient. Thus, the identification and the role of KRAS gene mutations in $\mathrm{mCRC}$ associated with the lack of response to targeted therapy with EGFR targeting $\mathrm{mAbs}$ is a game changer for the personalization of care. This includes biomarker discovery, patient selection, health economics, testing standardization, and quality assurance [52], and offers proof for the importance of additional factors apart from KRAS in influencing the course of disease [52-54], such as BRAF V600E [55].

- Taking the management of other non-mCRC liver metastases further: In personalizing the approach for MCRC we have to consider the high level of tumor heterogeneity in this type of 
cancer alone. This has to be multiplied by a significant factor when discussing the management of liver metastases derived from a variety of non-mCRC tumors. The only common factor in this setting is the liver as the anatomical site of occurrence. This fact on its own presents a considerable challenge in the evaluation of the disease and in guiding treatment in relation to the primary site. The clinical applications of personalized medicine in cancer and metastatic disease comprise screening, diagnosis, prognosis, prediction of response to treatment, followup after surgical or medical treatment, and early detection of disease progression. In addition, personalized medicine will guide patient stratification to therapies, including combination therapies with focused targeting for better efficacy and prevention of toxicity $[40,56]$.

Traditionally, commonly used techniques have focused on polymerase chain reaction, sequencing, immunohistochemistry, and fluorescent in situ hybridization. Now, following the completion of the Human Genome Project, the common tools for personalized medicine are high-throughput whole-genome sequencing, single nucleotide polymorphism haplotype mapping, microarray analysis, and proteomics/mass spectrometry and genome-wide association studies. These new techniques are integrated into a multiparametric approach and offer us the ability to improve sensitivity, specificity, and accuracy of new biomarkers [57].

Based on what we now know about the heterogeneity of cancer including intratumor heterogeneity and the cancer's dynamic response to selective pressure, mutational analysis of a single tumor biopsy is prone to underestimate the real mutational burden of highly heterogeneous tumors and their potential for resistance to therapy $[42,58,59]$. For this, testing a preparation of DNA/tumor 'cocktail' from multiple sites of disease may improve the yield of information and a better understanding of individual tumor biology.

The impact of quality and standardization in tissue acquisition and biobanking is a central precondition for generating highly reliable and reproducible results and data $[60,61]$. This enables a more precise assessment of the individual patient's disease. Additionally, with regards to the approach of metastatic disease, consideration should also be given to the difference between the primary and metastatic sites. Due to their evolution during the disease progression the genomic drivers may not always be evident in the primary tumor. These include the differences in the dysregulation of biomarker signaling as those observed in cases of mCRC [62]. Therefore, in the presence of metastatic disease, evaluation of the disease biology in both the primary and metastatic sites is warranted. Understanding the molecular mechanisms including those related to the response/resistance to treatment requires pairing clinical with biological information in a prospective manner, combining both clinical and functional validation of common and low frequency mutations occurring at diagnosis, and tracking their evolution over time. The need for predictive biomarkers to enable optimal treatment selection for each patient is thus increased. The application of PM technologies in these cases offers the opportunity for a dynamic approach required to investigate the mysteries of liver metastases and gain actionable, potentially clinically useful informa- tion for effective management. The dynamic testing over the course of therapy with repeated tumor biopsies upon progression or resistance may be crucial in understanding the individual tumor biology as well as unveiling its vulnerabilities and the mechanisms of resistance. Serial monitoring would provide the means on how molecular networks in disease respond to therapeutic 'challenges', thereby providing an insight into how they work [63]. Parallel analysis of biomarkers relevant to the primary tumor in the patients' serum including circulating tumor cells is warranted as sometimes these can be detected in advance of radiological progression $[64,65]$.

Dynamic monitoring of tumor biology during treatment could permit early initiation of combination treatment to prevent or delay progression, or a change of therapy to a more effective agent. Adapting a personalized approach in these cases would also assist in the evaluation and understanding of primary tumor biology in relation to the metastatic disease and also assist in identifying the drivers of carcinogenesis, hepatocarcinogenesis, biomarkers of response, and/or be predictive of the development of liver metastases. Of interest is the evaluation of strategies combining antiproliferative drugs with antiangiogenics to increase response and efficacy and to prevent metastases and or disease recurrence as antiangiogenesis targeting alone may have adverse effects, potentially promoting local invasion and metastatic spread [66].

The majority of advanced tumor types harbor biologically informative alterations. The use of techniques such as whole-exome sequencing (WES) in substitution of focused gene panel assays and in combination with prospective follow-up of patients integrating clinical with biological data can help in the identification of candidate predictive biomarkers of response [67]. Applying other methods and novel approaches such as hierarchical clustering to analysis of protein patterns in cancer-associated liver disease may create a basis for personalized treatment by providing insights into molecular mechanisms of drug clearance [68].

Another important factor to consider when dealing with complex, highly heterogeneous systems such as liver metastases is the design limitations imposed by the traditional models of evidencebased medicine and randomized clinical trials: Patient study populations are often empirically stratified into separate cohorts without a deeper understanding or analysis of the investigated disease. Stratification may only be driven by non-disease-related aspects of the clinical process. The assumption in this model is that the patients are sufficiently similar to allow valid comparison between the study groups, or in other words: 'one size fits all'. However, this model is insensitive in detecting biologically and clinically relevant signals because of noise attributable to the heterogeneity of the population examined [9].

In an effort to explore these complex and highly heterogeneous systems further, to distill, interpret, and contextualize meaningful genomic information of individual patients where no specific guidelines exist, a different approach is needed involving a novel clinical trial design. In the context of personalized medicine, clinical trials are likely to be smaller, involving a selected group of patients most likely to benefit from specific treatment or interven- 
tion. In this setting, patients can serve as their own controls (intraindividual control) [69].

In the past, we had to select clinical trial participants on the basis of a low frequency of molecular changes and statistics. NGS and gene expression profiling can be utilized to efficiently screen patients, to define prognosis, and to stratify to treatment entities. Beltran et al. [67] recently described an actual example of such an evidence-based clinical trial design using PM to understand how WES data affect clinical therapeutic decision making in patients with advanced metastatic cancer involving liver metastases and identified novel biomarkers of response. Unique aspects of this trial design included the analysis of more than 21,000 genes instead of a focused gene assay, complete disclosure of results through a WES clinical report, incorporation of metastatic site and serial biopsies, use of fresh frozen and formalin-fixed tissue, and development of patient-derived organoids and xenografts as a model for related clinical trials. In addition, a comprehensive computational pipeline was generated to categorize mutations and to compile a report for discussion in a multidisciplinary tumor board and clinical follow-up to assess the impact of detected mutations on subsequent responses to therapy and patient outcomes as integral parts of this trial.

In this proof of principle trial [67], the authors showed the ability to detect biologically informative genomic alterations in the vast majority of patients and were able to make treatment recommendations based on these findings in more than $90 \%$ of the cases. The ability for an actual change of treatment though was limited to only $5 \%$ of the cases due to limitations imposed on patient access to therapeutic trials. This kind of clinical trial design enables active instead of empirical stratification of patients. In addition, such designs offer the ability to capitalize on the information provided by omics technologies on the basis of active participation and involvement of patients in discovery-based clinical research. More importantly, such designs enable the development of an evidence base in parallel pace to that of genomic discoveries and therefore should result in a more rapid development of targeted therapies.

The vision of PM is based on the vast opportunity offered by the availability of new technologies and the success of current targeted therapies whose efficacies are predicted by certain biomarkers. However, the patients who can currently draw clinical benefit are overall still quite small. The question for us is: How can we turn this vision of PM into reality? For transforming this currently primarily informative approach into a practical clinical translation we need to address the still existing operational challenges regarding process, cost effectiveness, and regulatory and ethical issues/requirements [70].

\section{Conclusion}

Many aspects of PM have been in our daily practice since decades, and now the potential for new discoveries grows exponentially in the right settings. In an aim to fully exploit the opportunities which technology offers in addressing the complex problem of cancer, our approach to cancer as an entity and an area of research requires a reassessment, including a change of culture in medicine [9]. Adequate technologies around a well-defined clinical setting and an excellent biobanking strategy will enable the generation of comprehensive biological and molecular research results with tissue samples and liquid biopsies obtained from cancer patients.

The personalized approach in the management of nCRLMs is not a fiction. On the contrary: Unraveling the complexities of the biological behavior of tumors in this extremely heterogeneous setting can only be elucidated by increasingly embracing a personalized approach and the use of PM. As advanced technologies become available at decreasing costs, the use of precision oncology will provide significantly better information and tools to select patients, to define effective treatments, to minimize toxicity, and therefore to improve outcomes. Cancer management and therapeutics are currently undergoing a transition whereby the application of PM is rapidly becoming a reality in clinical practice.

\section{Disclosure Statement}

The authors declare no conflicts of interest.

\section{References}

1 Grammaticos PC, Diamantis A: Useful known and unknown views of the father of modern medicine, Hippocrates and his teacher Democritus. Hell J Nucl Med 2008;11:2-4.

2 Silver M: The Case for Personalized Medicine. Washington, DC, Personalized Medicine Coalition, 2009. www.personalizedmedicinecoalition.org/Userfiles/ PMC-Corporate/file/the_case_for_pm1.pdf.

3 Vesalius A: De humani corporis fabrica. San Francisco, Norman Publishing, 1998.

4 Virchow R: Die Cellularpathologie in ihrer Begründung auf physiologische und pathologische Gewebelehre: zwanzig Vorlesungen, gehalten während der Monate Februar, März und April 1858 im pathologischen Institute zu Berlin. Berlin, Hirschwald, 1858.
5 Chen R, Snyder M: Promise of personalized omics to precision medicine. Wiley Interdiscip Rev Syst Biol Med 2013;5:73-82.

6 Collins FS, Varmus H: A new initiative on precision medicine. N Engl J Med 2015;372:793-795.

Mirnezami R, Nicholson J, Darzi A: Preparing for precision medicine. N Engl J Med 2012;366:489-491.

8 NCI Dictionary of Cancer Terms: Personalized Medicine. www.cancer.gov/publications/dictionaries/cancerterms?cdrid=561717 (accessed October 10, 2015).

9 Blau CA, Liakopoulou E: Can we deconstruct cancer, one patient at a time? Trends Genet 2013;29:6-10.

10 Thariani R, Veenstra DL, Carlson JJ, Garrison LP, Ramsey S: Paying for personalized care: cancer biomarkers and comparative effectiveness. Mol Oncol 2012;6:260-266.
11 Allen TC: Payment for cancer biomarker testing. Arch Pathol Lab Med 2015;139:300-304.

12 Dzau VJ, Ginsburg GS, Van Nuys K, Agus D, Goldman $\mathrm{D}$ : Aligning incentives to fulfil the promise of personalised medicine. Lancet 2015;385:2118-2119.

13 Schork NJ: Personalized medicine: time for one-person trials. Nature 2015;520:609-611.

14 International HapMap Consortium: A haplotype map of the human genome. Nature 2005;437:1299-1320.

15 Frazer KA, Ballinger DG, Cox DR, et al: A second generation human haplotype map of over 3.1 million SNPs. Nature 2007;449:851-861.

16 Landsteiner K: Zur Kenntnis der antifermentativen, lytischen und agglutinierenden Wirkungen des Blutserums und der Lymphe. Zbl Bakt 1900;27:357-362. 
17 Heimburg-Molinaro J, Lum M, Vijay G, Jain M, Almogren A, Rittenhouse-Olson K: Cancer vaccines and carbohydrate epitopes. Vaccine 2011;29:8802-8826.

18 Old LJ: Cancer immunology: the search for specificity - G.H.A. Clowes Memorial lecture. Cancer Res 1981; 41:361-375.

19 Sahin U, Tureci O, Schmitt H, et al: Human neoplasms elicit multiple specific immune responses in the autologous host. Proc Natl Acad Sci U S A 1995;92:1181011813.

20 Callahan MK, Wolchok JD: Clinical activity, toxicity, biomarkers, and future development of CTLA-4 checkpoint antagonists. Sem Oncol 2015;42:573-586.

21 Overwijk WW, Wang E, Marincola FM, Rammensee $\mathrm{H}$, Restifo NP: Mining the mutanome: developing highly personalized immunotherapies based on mutational analysis of tumors. J Immunother Cancer 2013; $1: 1-4$.

22 Blaser MJ: The microbiome revolution. J Clin Invest 2014;124:4162-4165.

23 Ravel J, Blaser MJ, Braun J, et al: Human microbiome science: vision for the future, Bethesda, MD, July 24 to 26, 2013. Microbiome 2014;2:1-11.

24 Surace L, Lysenko V, Fontana AO, et al: Complement is a central mediator of radiotherapy-induced tumorspecific immunity and clinical response. Immunity 2015;42:767-777.

25 Zitvogel L, Kroemer G: Subversion of anticancer immunosurveillance by radiotherapy. Nat Immunol 2015 16:1005-1007.

26 Locher C, Rusakiewicz S, Tesniere A, et al: Witch hunt against tumor cells enhanced by dendritic cells. Ann N Y Acad Sci 2009;1174:51-60.

27 Zhang Y, Su HC, Lenardo MJ: Genomics is rapidly advancing precision medicine for immunological disorders. Nat Immunol 2015;16:1001-1004.

28 Moertel CG, Sauer WG, Dockerty MB, Baggenstoss AH: Life history of the carcinoid tumor of the small intestine. Cancer 1961;14:901-912.

29 Moertel CG: Karnofsky memorial lecture. An odyssey in the land of small tumors. J Clin Oncol 1987;5:15021522.

30 Thompson GB, van Heerden JA, Grant CS, Carney JA, Ilstrup DM: Islet cell carcinomas of the pancreas: a twenty-year experience. Surgery 1988;104:1011-1017.

31 Delcore R, Friesen SR: Gastrointestinal neuroendocrine tumors. J Am Coll Surg 1994;178:187-211.

32 Godwin JD: Carcinoid tumors an analysis of 2837 cases. Cancer 1975;36:560-569.

33 Sjoblom SM: Clinical presentation and prognosis of gastrointestinal carcinoid tumours. Scand J Gastroenterol 1988;23:779-787.

34 Choti MA, Bulkley GB: Management of hepatic metastases. Liver Transpl Surg 1999;5:65-80.

35 Bressac B, Kew M, Wands J, Ozturk M: Selective G to $\mathrm{T}$ mutations of p53 gene in hepatocellular carcinoma from southern Africa. Nature 1991;350:429-431.

36 Hsu I, Metcalf R, Sun T, Welsh J, Wang N, Harris C: Mutational hot spot in the p53 gene in human hepatocellular carcinomas. Nature 1991;350:427-428.

37 European Society of Radiology (ESR): Medical imaging in personalised medicine: a white paper of the research committee of the European Society of Radiology (ESR). Insights Imaging 2015;6:141-155
38 Bruix J, Han K, Gores G, Llovet JM, Mazzaferro V: Liver cancer: approaching a personalized care. J Hepatol 2015;62:S144-S156.

39 Venter JC: Multiple personal genomes await. Nature 2010;464:676-677.

40 Diamandis M, White NM, Yousef GM: Personalized medicine: marking a new epoch in cancer patient management. Mol Cancer Res 2010;8:1175-1187.

41 Curtis C, Shah SP, Chin S, et al: The genomic and transcriptomic architecture of 2,000 breast tumours reveals novel subgroups. Nature 2012;486:346-352.

42 Gerlinger M, Rowan AJ, Horswell S, et al: Intratumor heterogeneity and branched evolution revealed by multiregion sequencing. N Engl J Med 2012;366:883892.

43 Shibata D: Cancer. Heterogeneity and tumor history. Science 2012:336:304-305.

44 Marusyk A, Almendro V, Polyak K: Intra-tumour heterogeneity: a looking glass for cancer? Nature Reviews Cancer 2012;12:323-334

45 Gerlinger M, Swanton C: How Darwinian models inform therapeutic failure initiated by clonal heterogeneity in cancer medicine. Br J Cancer 2010;103:11391143.

46 Heinemann V, Douillard JY, Ducreux M, Peeters M: Targeted therapy in metastatic colorectal cancer - an example of personalised medicine in action. Cancer Treat Rev 2013;39:592-601.

47 O’Dwyer PJ, Catalano RB: Uridine diphosphate glucuronosyltransferase (UGT) $1 \mathrm{Al}$ and irinotecan: practical pharmacogenomics arrives in cancer therapy. J Clin Oncol 2006;24:4534-4538.

48 Cecchin E, Innocenti F, D’Andrea M, et al: Predictive role of the UGT1A1, UGT1A7, and UGT1A9 genetic variants and their haplotypes on the outcome of metastatic colorectal cancer patients treated with fluorouracil, leucovorin, and irinotecan. J Clin Oncol 2009;27: 2457-2465.

49 Ross JS, Torres-Mora J, Wagle N, Jennings TA, Jones DM: Biomarker-based prediction of response to ther apy for colorectal cancer: current perspective. Am J Clin Pathol 2010;134:478-490.

50 Weickhardt A, Wells K, Messersmith W: Oxaliplatininduced neuropathy in colorectal cancer. J Oncol 2011; 2011:201593.

51 Watanabe T, Kobunai T, Yamamoto Y, et al: Gene expression signature and response to the use of leucovorin, fluorouracil and oxaliplatin in colorectal cancer patients. Clin Transl Oncol 2011;13:419-425.

52 Bellon E, Ligtenberg MJ, Tejpar S, et al: External quality assessment for KRAS testing is needed: setup of a European program and report of the first joined regional quality assessment rounds. Oncologist 2011;16: $467-478$.

53 Wong R, Cunningham D: Using predictive biomarkers to select patients with advanced colorectal cancer for treatment with epidermal growth factor receptor antibodies. J Clin Oncol 2008;26:5668-5670.

54 Hawkes E, Cunningham D: Relationship between colorectal cancer biomarkers and response to epidermal growth factor receptor monoclonal antibodies. J Clin Oncol 2010;28:e529-531; author reply e532-e533.
Yang H, Higgins B, Kolinsky K, et al: Antitumor activity of BRAF inhibitor vemurafenib in preclinical models of BRAF-mutant colorectal cancer. Cancer Res 2012;72:779-789.

56 Diamandis EP, Li M: The side effects of translational omics: overtesting, overdiagnosis, overtreatment. Clin Chem Lab Med 2015;DOI: 10.1515/cclm-2015-0762.

57 Arsanious A, Bjarnason GA, Yousef GM: From bench to bedside: current and future applications of molecular profiling in renal cell carcinoma. Mol Cancer 2009; $8: 20$.

58 Richman SD, Chambers P, Seymour MT, et al: Intratumoral heterogeneity of KRAS and BRAF mutation status in patients with advanced colorectal cancer (aCRC) and cost-effectiveness of multiple sample testing. Anal Cell Pathol (Amst) 2011;34:61-66.

59 Garraway LA, Lander ES: Lessons from the cancer genome. Cell 2013;153:17-37.

60 Juhl H: Preanalytical aspects: a neglected issue. Scand J Clin Lab Invest Suppl 2010;242:63-65.

61 David KA, Unger FT, Uhlig P, et al: Surgical procedures and postsurgical tissue processing significantly affect expression of genes and EGFR-pathway proteins in colorectal cancer tissue. Oncotarget 2014;5:1101711028.

62 Molinari F, Martin V, Saletti P, et al: Differing deregulation of EGFR and downstream proteins in primary colorectal cancer and related metastatic sites may be clinically relevant. Br J Cancer 2009; 100:1087-1094.

63 Del Sol A, Balling R, Hood L, Galas D: Diseases as network perturbations. Curr Opin Biotechnol 2010;21: 566-571.

64 Diaz LA Jr, Williams RT, Wu J, et al: The molecular evolution of acquired resistance to targeted EGFR blockade in colorectal cancers. Nature 2012;486:537540.

65 Misale S, Yaeger R, Hobor S, et al: Emergence of KRAS mutations and acquired resistance to anti-EGFR therapy in colorectal cancer. Nature 2012;486:532-536.

66 Pàez-Ribes M, Allen E, Hudock J, et al: Antiangiogenic therapy elicits malignant progression of tumors to increased local invasion and distant metastasis. Cancer Cell 2009;15:220-231.

67 Beltran H, Eng K, Mosquera JM, et al: Whole-exome sequencing of metastatic cancer and biomarkers of treatment response. JAMA Oncol 2015;1:466-474.

68 Petushkova NA, Pyatnitskiy MA, Rudenko VA, et al: Applying of hierarchical clustering to analysis of protein patterns in the human cancer-associated liver. PLoS One 2014;9:e103950.

69 Von Hoff DD, Stephenson JJ Jr, Rosen P, et al: Pilot study using molecular profiling of patients' tumors to find potential targets and select treatments for their refractory cancers. J Clin Oncol 2010;28:4877-4883.

70 MacConaill LE, Lindeman NI, Rollins BJ: Brave-ish new world - what's needed to make precision oncology a practical reality. JAMA Oncol 2015;1:879-880. 